\title{
Determination of the Accuracy of 5 Electronic Apex Locators in the Function of Different Employment Protocols
}

\author{
Tiago Nepomuceno Oliveira, DDS, * Nilton Vivacqua-Gomes, DDS, MS, PbD, ${ }^{\dagger}$ \\ Ricardo Affonso Bernardes, DDS, MS, PhD, ${ }^{\neq}$Rodrigo Ricci Vivan, DDS, MS, PhD, ${ }^{\downarrow}$ \\ Marco Antonio Hungaro Duarte, DDS, MS, PbD, \\ and Bruno Carvalbo Vasconcelos, DDS, MS, $P b D^{7}$
}

\section{Ahstract}

Introduction: The aim of this study was to evaluate the accuracy of 5 electronic apex locators (EALs): Root ZX II (RZX; J Morita, Tokyo, Japan), Raypex 6 (RAY; VDW GmbH, Munich, Germany), Apex ID (AID; SybronEndo, Orange, CA), Propex II (PRO; Dentsply Maillefer, Ballaigues, Switzerland), and Propex Pixi (PIXI, Dentsply Maillefer) when used in the following protocols: (1) -1.0 , insertion up to $1.0 \mathrm{~mm}$ below the apical foramen (AF); (2) 0.0/-1.0, insertion until the AF and withdrawn $1.0 \mathrm{~mm}$ short of the AF; (3) 0.0 , insertion until the AF; and (4) over/0.0, insertion until "over" and withdrawal to AF. Methods: Thirty human lower premolars had coronary accesses and cervical and middle thirds preparations performed, allowing AF standardization $(200 \mu \mathrm{m})$. Using an alginate experimental model, root canal length $(\mathrm{RCL})$ measurements were performed sequentially with EALs following each of the protocols. Results: Considering the suggested protocols, the lowest mean error values were observed in $0.0,0.10 \mathrm{~mm}(\mathrm{RZX}), 0.13 \mathrm{~mm}$ (RAY), $0.16 \mathrm{~mm}$ (AID), $0.23 \mathrm{~mm}$ (PRO), and $0.10 \mathrm{~mm}$ (PIXI), without a significant difference for over/0.0 $(P>.05)$. Comparing the results obtained in 0.0 with those found in -1.0 and $0.0 /-1.0$, significant differences were observed for most EALs $(P<.05)$. For the comparison between EALs, significant differences were observed only in protocols -1.0 and over/0.0 $(P<.05)$. Conclusions: Under the conditions of the study, it was concluded that, regardless of the mechanism of the device, the best results were found when electronic RCL measurement was performed at the AF; furthermore, the electronic withdrawal did not offer any additional advantage over the reach of the AF. (J Endod 2017;43:1663-1667)

\section{Key Words}

Electronic apex locators, measurement protocol, root canal length

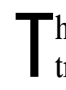
he evolution of electronic apex locators (EALs) has reached accuracy levels above $95 \%$ depending on the conditions in which they are used (1-6). These clinical conditions are directly related to the apical fit of the instrument $(2,7,8)$, the apical limit of the determination $(7,9)$, the impossibility of foraminal patency (9), the dynamics of the electronic determinations $(5,10,11)$, and so on $(12-15)$. Several studies have been dedicated to evaluate the accuracy of EALs, and in these, different employment protocols have been used offering conflicting results regarding the accuracy of electronic devices. Protocols that determine the insertion of the instruments until they reach some anatomic position (eg, the apical foramen [AF], apical constriction, or even positions below the AF) $(7,9,11,16)$ are confronted with those that perform the insertion of the instruments until the AF followed by withdrawal to a position determined in the device display $(6,14)$.

Since the study of Kobayashi and Suda (17), which represented the introduction of the mechanism of operation of EALs based on the principle of frequencydependent impedance, EALs have been trusted with the fundamental role in determining the root canal length (RCL). However, even using the same principle, the currently available EALs operate mechanisms that differ from the originally proposed ones. Some use the impedance quotient principle such as Root ZX II (0.4 and $8 \mathrm{kHz}$ ) (J Morita, Tokyo, Japan) (15) and Apex ID (0.5 and $5 \mathrm{kHz}$ ) (SybronEndo, Orange, CA) (9). Raypex 6 (0.4 and $8 \mathrm{kHz}$ ) (VDW GmbH, Munich, Germany) (13), Propex II $(0.5$ and $8 \mathrm{kHz})$ (Dentsply Maillefer, Ballaigues, Switzerland) (18), and the recently launched Propex Pixi (Dentsply Maillefer) (14) use the square root of impedances in 2 frequencies. Other EALs currently available use other mathematical reasons between impedance values in 2 or more frequencies measured simultaneously or separately (1). These mechanisms of impedance interpretation

From the *Private Practice, Fortaleza, Ceará, Brazil; ${ }^{\dagger}$ School of Dentistry of Ceará, São Leopoldo Mandic University, Fortaleza, Ceará, Brazil; ${ }^{\ddagger}$ Brazilian Dental Association, Taguatinga, Distrito Federal, Brazil; ${ }^{\S}$ Department of Dentistry, Endodontics and Dental Materials, Bauru Dental School, University of São Paulo, Bauru, São Paulo, Brazil; and "School of Dentistry of Sobral, Federal University of Ceará, Sobral, Ceará, Brazil.

Address requests for reprints to Dr Bruno Carvalho Vasconcelos, School of Dentistry of Sobral, Federal University of Ceará, Campus Sobral, CEP 62010-590, Sobral, CE, Brazil. E-mail address: bcv@ufc.br

0099-2399/\$ - see front matter

Copyright () 2017 American Association of Endodontists. http://dx.doi.org/10.1016/j.joen.2017.03.039 
have been considered as possible determinants of the accuracy of EALs in different clinical conditions of use $(1,9)$.

Considering the importance of the correct determination of RCL in the success of endodontic treatment (19), the present study aimed to evaluate the role of the electronic RCL protocols in the accuracy of the Root ZX II, Raypex 6, Apex ID, Propex II, and the recently launched Propex Pixi EALs, evaluating the following protocols: \#1 $(-1.0)$, insertion up to $1.0 \mathrm{~mm}$ short of the AF; \#2 (0.0/-1.0), insertion until the AF and withdrawal until -1.0 ; \#3 $(0.0)$, insertion until reaching the AF; and \#4 (over/0.0), insertion until it surpasses the AF and then withdrawal to the AF. The null hypothesis considered was that no significant differences would be found between the electronic locators and the protocols tested.

\section{Methods}

The sample calculation was performed using G*Power v3.1 for Mac (Heinrich Heine, Universität Düsseldorf, Düsseldorf, Germany) using the Wilcoxon-Mann-Whitney test. Data from a previous study were considered, and the effect size in the present study was established (9). The alpha-type error of 0.05 at a beta power of 0.80 and an $N 2 / N 1$ ratio of 1 were also stipulated. A total of 30 samples were indicated as the ideal size required for noting significant differences.

After approval by the research ethics committee, 30 human lower premolar teeth extracted for orthodontic purposes were collected. The inclusion criteria considered included the need to present single channels (Vertucci type I) with patent AFs and teeth presenting length $<19 \mathrm{~mm}$ or $>21 \mathrm{~mm}, \mathrm{AF}>200 \mu \mathrm{m}$, curvature $>20^{\circ}$, and root resorption or caries; teeth that did not fulfill the aforementioned criteria were replaced.

The teeth had their coronary accesses made in a standardized way with diamond tips (\#1013 and \#3081; KG Sorensen, Cotia, SP, Brazil) that were operated in high rotation under abundant refrigeration. Also, wear was performed at the tips of cusp as a way to create flat references for positioning of the limiters of penetration of the instruments. C-Pilot manual files (\#10 and \#15, VDW GbmH) were used for the negotiation of the canals, after which the coronal and middle thirds were prepared with ProTaper S1 and Sx instruments (Dentsply Maillefer) inserted apically up to $5.0 \mathrm{~mm}$ short of the apparent canal length. After preflaring, the AFs were standardized with K-Flex file \#25 (Dentsply Maillefer) manual files using $2.5 \%$ sodium hypochlorite as an irrigating solution (Biodinâmica, Ibiporã, PR, Brazil).

The teeth were then randomly assigned into 5 groups of 6 teeth each for electronic RCL measurement; after being adapted to a special support, the teeth's apical portions were immersed in newly manipulated alginate (Jeltrate II; Dentsply, Petrópolis, RJ, Brazil). A lip clip was inserted into the alginate; all determinations were performed on fresh alginate ( $<30$ minutes). All the devices electronically measured the entire sample $(N=30)$.

The EALs were always used with maximum load on their drums or with new batteries. A single experienced endodontist who previously calibrated the EALs performed all the electronic RCLs in triplicate with manual instruments adjusted to the desired apical limit; the readings were recorded after 5 seconds of stability. The order of use of the EALs followed an alternate sequence to allow all of the equipment to be used the same number of times as the first one; its use followed 1 of the 4 protocols proposed.

\section{Protocol \#1 [-1.0]: Insertion up to $\mathbf{1 . 0} \mathbf{~ m m}$ Short of AF}

Each device had 1 pole attached to the labial loop and the other to a manual file that was in the middle third of the canal. The instrument was then inserted in an apical sense until the device's display showed the arrival of the instruments at position -1.0. At this time, the adjustment of the instrument was verified; in case of a mismatch, the procedure was repeated with a larger instrument. The Root ZX II device allows the indicator bar to be positioned at a point between 0.0 and 1.0 (the green indicative zone), and 1.0 was used in this protocol. Raypex 6 displays a graphic representation of positions and colors; the activation of the third green light-emitting diode (LED) was considered for determining the effect at position -1.0. The Apex ID and Propex II devices display this position both graphically and numerically ("1.0"). The Propex Pixi has an LED that represents this position.

\section{Protocol \#2 [0.0/-1.0]: Insertion to the AF and Withdrawal to -1.0 of the Device}

The electronic RCL was performed in a similar way to that previously described; however, this time, the manual instruments were inserted until the devices indicated the arrival at the AF. As described in the previous protocol, all the devices allow the identification of the arrival to the desired position; in the case of Root ZX II, it allows the calibration of its indicator bar to the 0.0 position. The Raypex 6 indicates the AF position with the activation of the red LED. On the Apex ID and Propex II devices, this information is provided on their displays (" 0.0 " and Apex). In turn, the Propex Pixi has an LED that represents the AF position (0.0). After confirming the adjustment of the instruments, the files were withdrawn coronally until the EALs indicated the references relative to the -1.0 position as described in the previous protocol.

\section{Protocol \#3 [0.0]: Insertion until Af}

The protocol used in this respect proceeded in the sequence previously described until reaching the AF; however, this time, the maintenance of the electronic determination and due registration was performed at the same level (AF).

\section{Protocol \#4 [over/0.0]: Insertion to Beyond the AF and Withdrawal}

In this protocol, the same sequence described earlier was followed for reaching the AF; however, this time it was overtaken, and the $\mathrm{AF}$ patency was appropriately pointed out by each of the electronic devices. The EAL Root ZX II indicates the arrival in the periapical region by activating the purple indicator bars. In turn, the Raypex 6 lights a red dot beyond the tooth illustration. Apex ID, Propex II, and Propex Pixi have an "over" position on their displays that presents a position beyond the AF. After the electronic validation of the patency, the manual instruments were withdrawn in a coronary direction until they indicated the arrival at the AF as previously described.

Regardless of the protocol used, after reading maintenance, the instruments had their rubber stops properly adapted to the occlusal references of the teeth. Then, they were carefully removed from the root canal and their lengths measured in a digital caliper $(0.001 \mathrm{~mm}$; Multitoyo, Suzano, SP, Brazil). After determining the penetration lengths, the last file used in each tooth was then reinserted in the canals until the extension of their stops and glued to the teeth with cyanoacrylate bond (Super Bonder; Loctite, São Paulo, SP, Brazil).

The teeth then had the apical $5.0 \mathrm{~mm}$ of their roots worn longitudinally in the buccal-lingual direction to allow the realization of $25 \times$ magnification digital photographs. The images captured were analyzed using ImageJ software (National Institutes of Health, Bethesda, MD) regarding the mistake offered by the devices; the RCLs were estimated on the basis of the sum of the measured errors in the software, positive or negative, and the extent of penetration by the fixed instrument. Considering the RCL, positive and negative values were attributed for readings beyond and below the target $(0.0 \mathrm{~mm}$ and $-1.0 \mathrm{~mm})$, respectively. The mean errors were calculated comparing the RCL determined by each device and the real RCL determined via the software. 
The tabulated data were then analyzed for normality (Shapiro-Wilk test), and the nonparametric nature of the data was defined according to the statistical analysis performed by the Kruskal-Wallis and Dunn tests, both using a level of significance of $5.0 \%$.

\section{Results}

Table 1 presents the median of the errors provided by the devices in each of the protocols. Considering the tested variations, the statistical analysis pointed out that, regardless of the EAL, the best results were observed when the devices had their penetration interrupted in the $\mathrm{AF}$ and, in this position, had their measurement determined. No significant differences were found between protocols \#3 (0.0) and \#4 (over/ $0.0)$ regardless of the device $(P>.05)$. However, when comparing the results found in these with those in protocols \#1 $(-1.0)$ and \#2 $(0.0 /$ $-1.0)$, significant differences were found in most EALs $(P<.05)$, except for Propex II and Propex Pixi in which no differences were found between them in protocols \#1 $(-1.0)$ and \#4 (over/0.0) $(P>.05)$.

Taking into account the results offered by the EALs in each of the protocols, it was observed that significant differences between the devices were observed only in protocols \#1 (-1.0) and \#4 (over/0.0). When the EALs performed the electronic RCL interrupting its penetration $1.0 \mathrm{~mm}$ short of the AF $(-1.0)$, significant differences were observed between Propex II and Raypex $6(P<.05)$, the EALs that offered the best and the worst results, respectively. When the electronic determinations were performed following protocol \#4 (over/0.0), the best results were observed with Root ZX II, Raypex 6, and Apex ID. A significant difference was found between them and Propex II $(P<.05)$, which offered the worst results in this condition.

Table 2 presents the accuracy percentages of the devices considering a tolerance range of $\pm 0.5 \mathrm{~mm}$. Similar to that observed for mean errors, the highest precision percentages were found in protocol \#3 (0.0), with the EAL precision ranging from $83 \%$ (Propex II) to $93 \%$ (Root ZX II, Raypex 6, Apex ID, and Propex Pixi). The lowest precision values were also observed in protocol \#2 $(0.0 /-1.0)$ in which the lowest precision was found for an EAL (Apex ID, 34\%).

Electronic RCL measurements beyond the AF were observed in all protocols, even those that aimed to determine the length $1.0 \mathrm{~mm}$ short of the AF; however, in this condition, they were much less frequent, occurring between $0.0 \%$ and $3.3 \%$. In the determinations performed at the AF level, the same occurred in all of the EALs; the lowest frequency was observed with Propex II in protocol \#3 (0.0) (13.3\%) and with Apex ID in protocol \#4 (over/0.0) (10\%).

\section{Discussion}

The present study evaluated the accuracy of 5 EALs when performing RCL measurements following different employment protocols. The accuracy of Root ZX II, Raypex 6, Apex ID, Propex II, and the recently introduced Propex Pixi in measurements performed $1.0 \mathrm{~mm}$ short of the $\mathrm{AF}(-1.0)$ and on it (0.0) was evaluated; however, up to the present, no similar study in literature has evaluated the accuracy of these devices when performing electronic withdrawal during the length determinations. In this way, electronic measurements were performed with instruments inserted until reaching the $\mathrm{AF}$ and then withdrawing them until $1.0 \mathrm{~mm}$ below it $(0.0 /-1.0)$ and in a condition in which the instruments were inserted until they passed through the AF and then were withdrawn until they reached it (over/0.0).

For this, an ex vivo research model was performed using alginate as an electroconductive medium. This model has been extensively used in the literature $(8,12,20-22)$ and has presented results that can be extrapolated to clinical conditions $(21,22)$. To eliminate methodological biases, only mandibular premolars of close lengths ( $>19 \mathrm{~mm}$ and $<21 \mathrm{~mm}$ ) with a single root canal (Vertucci type I) and with patent and standardizable AFs were used. To favor the electronic determinations, the coronal and middle thirds of the canals were previously preflared, and the instruments were adapted to the apical portion of the root canals at the moment of the procedure $(7,8,16)$.

The results found here highlight the excellent accuracy of the electronic determination devices when taken up to the AF after coronal preparation and using adjusted files. In the condition provided by protocol \#3 (0.0), the lowest mean error values $(0.1-0.23 \mathrm{~mm})$ and the highest precision percentages $(83 \%-93 \%)$ were found regardless of the operating mechanism. This finding corroborates with the literature that points to this as the ideal clinical condition for performing electronic RCL measurements and also that the different mechanisms used by the EALs have equal precision $(5,7-9,16)$.

The comparison of these findings with those obtained in protocol \#4 (over/0.0) indicates that, although not significant, with the exception of the Apex ID, all of the EALs offered poorer results than those produced when simply brought to the AF (0.0). As far as we know, such a comparison is not available in the literature. However, it is probable that this precision decrease is caused by the slight mismatch of the file upon its return to the coronary position; in this case, the file will be slightly misadjusted, influencing the capacitive factor that makes up the impedance determined by the devices $(1,9,11)$. However, the fact that it was performed at the AF level seems to favor the resistive factor because the results found are, for most EALs, higher than those observed in determinations performed shortly before the AF. In protocol \#3 (0.0), only Propex II offered significantly lower results than the other devices, highlighting some difficulty of understanding the electronic parameters in this circumstance.

Taking into account the determination performed according to protocol \#1 (-1.0) (ie, $1.0 \mathrm{~mm}$ below the AF), it was observed that the accuracy of the EALs was significantly smaller than those observed in the determinations performed at the $\mathrm{AF}$ level; the mean errors ranged from

TABLE 1. Median of the Errors (mm) Provided by Each Device's Electronical Measurements in Different Protocols Used

\begin{tabular}{lcccc}
\hline Device & $\begin{array}{c}\text { Insertion up to }-1.0 \\
\text { from the AF }(-1.0)\end{array}$ & $\begin{array}{c}\text { Insertion up to the } \\
\mathrm{AF} \text { and withdrawn } \\
\text { to }-1.0(0.0 /-1.0)\end{array}$ & $\begin{array}{c}\text { Insertion up to } \\
\text { the } A F(0.0)\end{array}$ & $\begin{array}{c}\text { Insertion up to beyond } \\
\text { the } A F \text { and withdrawn } \\
\text { to it (over/0.0) }\end{array}$ \\
\hline Root ZX II & $0.38^{\mathrm{ab}, \mathrm{B}}$ & $0.50^{\mathrm{a}, \mathrm{B}}$ & $0.10^{\mathrm{a}, \mathrm{A}}$ & $0.15^{\mathrm{a}, \mathrm{A}}$ \\
Raypex 6 & $0.66^{\mathrm{b}, \mathrm{B}}$ & $0.57^{\mathrm{a}, \mathrm{B}}$ & $0.13^{\mathrm{a}, \mathrm{A}}$ & $0.14^{\mathrm{a}, \mathrm{A}}$ \\
Apex ID & $0.36^{\mathrm{ab}, \mathrm{B}}$ & $0.71^{\mathrm{a}, \mathrm{B}}$ & $0.16^{\mathrm{a}, \mathrm{A}}$ & $0.13^{\mathrm{a}, \mathrm{A}}$ \\
Propex II & $0.31^{\mathrm{a}, \mathrm{A}}$ & $0.42^{\mathrm{a}, \mathrm{A}}$ & $0.23^{\mathrm{a}, \mathrm{A}}$ & $0.29^{\mathrm{b}, \mathrm{A}}$ \\
Propex pixi & $0.34^{\mathrm{ab}, \mathrm{AB}}$ & $0.42^{\mathrm{a}, \mathrm{AB}}$ & $0.10^{\mathrm{a}, \mathrm{A}}$ & $0.23^{\mathrm{ab}, \mathrm{AB}}$ \\
\hline
\end{tabular}

$\mathrm{AF}$, apical foramen.

The median was calculated in terms of absolute values of the determinations. Different superscript lowercase letters indicate statistically significant differences between devices according to the Kruskal-Wallis and Dunn tests $(P<.05)$. Different superscript uppercase letters indicate statistically significant differences between the measurements provided by devices considering each protocol according to the Kruskal-Wallis and Dunn tests $(P<.05)$. 
TABLE 2. The Accuracy of Apex Locator Determinations Provided in Each Protocol Considering a Tolerance Margin of $\pm 0.5 \mathrm{~mm}$

\begin{tabular}{|c|c|c|c|c|c|c|c|c|}
\hline \multirow[b]{2}{*}{ Device } & \multicolumn{2}{|c|}{$\begin{array}{l}\text { Insertion up to }-1.0 \\
\text { from the } A F\end{array}$} & \multicolumn{2}{|c|}{$\begin{array}{l}\text { Insertion up to the } A F \\
\text { and recede to }-1.0\end{array}$} & \multicolumn{2}{|c|}{ Insertion up to the AF } & \multicolumn{2}{|c|}{$\begin{array}{l}\text { Insertion up to beyond } \\
\text { the } A F \text { and recede to it }\end{array}$} \\
\hline & $n$ & $\%$ & $n$ & $\%$ & $n$ & $\%$ & $n$ & $\%$ \\
\hline Root ZX II & 18 & 60 & 15 & 50 & 28 & 93 & 28 & 93 \\
\hline Raypex 6 & 12 & 40 & 12 & 40 & 28 & 93 & 27 & 90 \\
\hline Apex ID & 21 & 70 & 10 & 33 & 28 & 93 & 28 & 93 \\
\hline Propex II & 20 & 67 & 15 & 50 & 25 & 83 & 19 & 63 \\
\hline Propex Pixi & 19 & 63 & 20 & 67 & 28 & 93 & 26 & 87 \\
\hline
\end{tabular}

$\mathrm{AF}$, apical foramen.

$0.31 \mathrm{~mm}$ (Propex II) to $0.66 \mathrm{~mm}$ (Raypex 6) and the accuracy from $70 \%$ (Apex ID) to 40\% (Raypex 6). Considering that the devices are exposed to a clear restriction in the resistive factor by not reaching the AF, the EALs presented different behaviors; Raypex 6 greatly decreased its accuracy, unlike Propex II, which did not suffer significant interference from this clinical condition. This finding has already been highlighted in previous studies that pointed out that distinct mechanisms of functioning interact differently in the face of clinical difficulties $(2,9,23)$.

All EALs display numbers, which, according to the manufacturers, do not represent the position of the files inserted in the canals; however, most operating manuals advise that professionals must interrupt the insertion of the instruments up to the " 0.5 " point or until they reach the AF and then withdraw them to this " 0.5 " point. This statement seems a clear reference to the location of the apical constriction determined by Kuttler (24). Previous studies have highlighted the advantages of RCL measurements performed at " 0.0 " (AF) rather than " 1.0 " $(5,7,9,11)$; however, until present, none have compared the accuracy of these devices in protocols that perform electronic withdraws. Following the same reasoning, the possibility of accomplishment of these retrocedes was evaluated using protocol \#2 $(0.0 /-1.0)$ in which the $\mathrm{AF}$ was reached and then the file lengths were retracted up to $1.0 \mathrm{~mm}$ short of it considering the device displays. For some authors, when reaching the AF level, the exact determination of the RCL would have been achieved; thus, the electronic withdrawal would have a solid electronic base to be performed. Apparently such reasoning is reproducible once the mean error and accuracy values found were worse than the others, even upon reaching the AF. This is probably related to the speed at which the electronic devices make their determinations, not considering in their displays an evolution of the reading of the position of the instruments inside the canals but rather the current position determined according to the clinical conditions at each moment.

The results found here point to the inaccuracy of EALs whenever the electronic withdrawal was performed $(-1.0 \times 0.0 /-1.0 ; 0.0 \times$ over/0.0). This is probably caused by the already mentioned fact that when advancing the instrument and withdrawing it, it will no longer be properly adjusted to the walls of the canals, an occurrence that has been pointed out as a cause of impairment in the interpretation of the capacitance factor of the impedance determined by the devices $(1,9,11,23)$. These findings do not corroborate with those of Piasecki et al (6), who observed a similarity between the electronic withdrawal and the one performed considering an electronic RCL measurement performed at the AF. This divergence could be attributed to methodological variations because these authors did not use adapted files during the determinations, which may have balanced the results.

Thus, it can be considered that the clinical indication for performing electronic RCL determinations is its execution at the AF level, reaching 0.0 or the "apex" of the devices. Such a protocol provides conditions for EALs, regardless of their operating mechanism, to correctly interpret local electronic parameters at the moment of measurement. The realization of electronic withdrawals as a way of providing an electronical reference for the devices with regard to the position of the AF did not really provide any increase in precision. Thus, it is not considered as advantageous. It is suggested that the establishment of the apical limit of instrumentation and/or root canal filling must be performed based on the determined RCL up to the foraminal limit.

\section{Conclusion}

This study found that the EALs offer their best results when the electronic determinations are performed up to the AF without surpassing it; in this case, there were no differences between the evaluated devices. Furthermore, we discovered that electronic withdrawal based on determinations performed at the AF or beyond it does not provide good results, negatively affecting the accuracy of the electronic devices.

\section{Acknowledgments}

Supported in part by FAPESP (\#2016/00245-1).

The authors deny any conflicts of interest related to this study.

\section{References}

1. Nekoofar MH, Ghandi MM, Hayes SJ, Dummer PM. The fundamental operating principles of electronic root canal length measurement devices. Int Endod J 2006;39: 595-609.

2. Akisue E, Gratieri SD, Barletta FB, et al. Not all electronic foramen locators are accurate on teeth with enlargement apical foramina: an in vitro comparison of 5 brands. J Endod 2014;40:109-12.

3. Martins JN, Marques D, Mata A, Carames J. Clinical efficacy of electronic apex locators: systematic review. J Endod 2014;40:759-77.

4. Khandewal D, Ballal NV, Saraswhati MV. Comparative accuracy of 2 electronic apex locators with conventional radiograph: an ex vivo study. J Endod 2015;41:201-4.

5. Parente LA, Levin MD, Vivan RR, et al. Efficacy of electronic foramen locators in controlling root canal working length during rotary instrumentation. Braz Dent J 2015; 26:547-51.

6. Piasecki L, Carneiro E, da Silva Neto UX, et al. The use of micro-computed tomography to determine the accuracy of 2 electronic apex locators and anatomic variations affecting their precision. J Endod 2016;42:1263-7.

7. Stool R, Urban-Klein B, Roggendorf MJ, et al. Effectiveness of four electronic apex locators to determine distance from apical foramen. Int Endod J 2010;43:808-17.

8. Vasconcelos BC, Matos LA, Pinheiro-Júnior EC, et al. Ex vivo accuracy of three electronic apex locators using different apical file sizes. Braz Dent J 2012;23:199-204.

9. Vasconcelos BC, Verissimo-Chavez RD, Vivacqua-Gomes N, et al. Ex vivo evaluation of the accuracy of electronic foramen locators in root canals with an obstructed apical foramen. J Endod 2015;41:1551-4.

10. Wigler R, Huber R, Lin S, Kaufman AY. Accuracy and reliability of working length determination by Gold Reciproc motor in reciprocating movement. J Endod 2014; $40: 694-7$.

11. Vasconcelos BC, Frota LM, Souza T, et al. Evaluation of the maintenance of the apical limit during instrumentation with hybrid equipment in rotary and reciprocating modes. J Endod 2015;41:682-5.

12. Gehlot PM, Manjunath V, Manjunath MK. An in vitro evaluation of the accuracy of four electronic apex locators using stainless-steel and nickel-titanium hand files. Restor Dent Endod 2016;41:6-11.

13. Marigo L, Gervasi GL, Somma F, et al. Comparison of two electronic apex locators on human cadavers. Clin Oral Investig 2016;20:1547-50. 
14. Üstün Y, Aslan T, Sekerci AE, Sağsen B. Evaluation of the reliability of cone-beam computed tomography scanning and electronic apex locator measurements in working length determination of teeth with large periapical lesions. J Endod 2016; $42: 1334-7$.

15. Altunbas D, Kustarci A, Toyoglu M. The Influence of various irrigants on the accuracy of 2 electronic apex locators in locating simulated root perforations. J Endod 2017;43:439-42.

16. Camargo EJ, Zapata RO, Medeiros PL, et al. Influence of preflaring on the accuracy of length determination with four electronic apex locators. J Endod 2009;35: $1300-2$.

17. Kobayashi C, Suda H. New electronic canal measuring device based on the ratio method. J Endod 1994;20:111-4.

18. Riccuci D, Langeland K. Apical limit of root canal instrumentation and obturation, part 2. Histological study. Int Endod J 1998;31:394-409.
19. Cianconi L, Angotti V, Felici R, et al. Accuracy of three electronic apex locators compared with digital radiography: an ex vivo study. J Endod 2010;36:2003-7.

20. Baldi JV, Victorino FR, Bernardes RA, et al. Influence of embedding media on the assessment of electronic apex locators. J Endod 2007;33:476-9.

21. Duran-Sidreu F, Stober E, Mercandé M, et al. Comparison of in vivo and in vitro readings when testing the accuracy of the Root $\mathrm{ZX}$ apex locator. J Endod 2012;38:236-9.

22. Soares RM, Silva EJ, Herreira DR, et al. Evaluation of Joypex 5 and Root ZX II: an in vivo and ex vivo study. Int Endod J 2013;46:904-9.

23. Vasconcelos BC, Bueno MM, Luna-Cruz SM, et al. Accuracy of five electronic foramen locators with different operating systems: an ex vivo study. J Appl Oral Sci $2013 ; 21: 132-7$.

24. Kuttler Y. Microscopic investigation of root apexes. J Am Dent Assoc 1955;50: 544-52. 